\title{
Jenseits der biographischen Illusion?
}

\author{
Neuere Biographik in Wissenschafts- und Medizingeschichte
}

\section{Christoph Gradmann}

Dehli, Martin, 2007. Leben als Konflikt. Zur Biographie Alexander Mitscherlichs. Göttingen: Wallstein Verlag, geb. 320 S., 29,90€, ISBN-13:978-3835300637.

Freimüller, Tobias, 2007. Alexander Mitscherlich. Gesellschaftsdiagnose und Psychoanalyse nach Hitler. Göttingen: Wallstein Verlag, geb. 480 S., 39,90 €, ISBN-13: 978-3835301870.

Linton, Derek S., 2005. Emil von Behring. Infectious Disease, Immunology, Serum Therapy. Philadelphia: American Philosophical Society, geb. 580 S., 65 \$, ISBN-10: 0871692554.

Söderqvist, Thomas, Hg., 2007. The History and Poetics of Scientific Biography. Aldershot: Ashgate, geb. 300 S., 114,95 \$, ISBN-13: 978-0754651819.

Zigman, Peter, Hg., 2006. Die biographische Spur in der Kulturund Wissenschaftsgeschichte. Jena: Edition Paideia, brosch. $258 \mathrm{~S}$., $33 €$, ISBN-13: 978-3938203453.

Betrachtet man die Entwicklung der Geschichtswissenschaft in den letzten zwanzig Jahren, so gehört die Rehabilitierung der historischen Biographie zu den auffälligen Phänomenen. Das in den 1970er Jahren als „letzte Auffangstellung des Historismus" (Oelkers 1974: 299) gebrandmarkte Genre stand seinerzeit im Verdacht des methodisch Ewiggestrigen. In einem der tonangebenden Bücher der Zeit, Hans Ulrich Wehlers Das Deutsche Kaiserreich 1914-1918, wurde, so ein Rezensent ironisch, der „Wilhelminismus ohne 
Wilhelm, das Kaiserreich ohne Kaiser" (Sombart 1978) analysiert. Es schadete dem Ansehen des Buches nicht. Genauso - ohne Wilhelm also - war das Kaiserreich seinerzeit am besten zu verstehen, darf man mithin annehmen. Kennzeichen der Epoche war das Desinteresse am biographischen Zugang, und nur wenigen Autoren erschien - wie Fritz Stern in seiner Parallelbiographie Bismarcks und seines Bankiers Bleichröder (1988 [1977]) - moderne Sozial- und Mentalitätsgeschichte mit der Form der Biographie vereinbar. ${ }^{1}$

Demgegenüber scheint die Biographie mittlerweile wieder im Zentrum der Geschichtswissenschaft angekommen: Margit Szöllösi Janze erhielt 1998 für ihre Biographie Fritz Habers den Preis des deutschen Historikerverbands. Als Buch des Jahres 2002 zeichnete das Internet Journal H-Soz-Kult (in der offenen Kategorie) die Autobiographie des britischen Historikers Eric Hobsbawm aus, und Jan Eckels intellektuelle Biographie Hans Rothfels' von 2005 wurde allgemein als wichtiger Beitrag eben nicht nur zur Biographik, sondern zur Wissenschaftsgeschichte der Historiographie selbst angesehen. Genauso wenig fehlt es an Sammelbänden, die sich auf die Methodik des Genres beziehen (Boedeker 2003, Klein 2002). Die Gründe für eine derartige Renaissance sind sicher vielfältig, aber zwei erscheinen besonders wichtig. Zum einen fand die Skepsis der Fachleute zu keiner Zeit den Weg ins Publikum. Auf die Dauer kann es sich ein so öffentliches Fach wie die Geschichtswissenschaft nicht leisten, die Leserschaft jenseits der eigenen Fachkollegen zu ignorieren. Zum anderen blieb die Kritik am eindimensionalen Persönlichkeitsbegriff der traditionellen Biographik nicht ohne Wirkung. Dass eine historische Persönlichkeit kein natürliches Objekt, sondern ein mithilfe zumeist soziologischer Theorie und historischer Narration hergestellter Gegenstand ist, gehört zu den Grundbegriffen moderner Biographik. Ausgerechnet Pierre Bourdieus La illusion biographique (1990), eine radikale Kritik des kohärenten Persönlichkeitsbegriffes, wurde auf diese Weise zu einem Schlüsseltext, der in vielen neueren Biographien zur Diskussion der Methode herangezogen wird.

Für die Wissenschaftsgeschichte gilt Ähnliches: Lange Zeit erschien die Biographik als das übelste Beispiel jener "bad old history of science [...], which we have all been told to abhor" (Hankins 1979: 2). Diese schlechte alte Biographik ist durchaus immer noch vorhanden: in Kurz- und Kürzestbeiträgen in naturwissenschaftlichen oder medizinischen Fachzeitschriften oder in der von Robert Merton so genannten Eponymie (Merton 1985), also der leicht zwanghaft anmutenden Sitte, in populären Handbüchern historische Ereignisse wenn immer möglich mit einem Namensetikett zu versehen. Auffällig ist auch die große Zahl sozusagen tertiärliterarischer Biographik: Eine vor einigen Jahren erschienene Biographische Enzyklopädie deutschsprachiger Mediziner etwa entpuppt sich bei näherer Betrachtung schlicht als ein Auszug aus der Deutschen Biographischen Enzyklopädie. Zu nennen ist schließlich auch das Internet. In der freien Enzyklopädie Wikipedia sind, 
nach dem persönlichen Eindruck des Verfassers, biographische Artikel zumeist im heroischen Duktus gehalten und manchmal auch biographische Details, ja gelegentlich die ganze Person frei erfunden (Müller 2008). Dass Wissenschafts-, Medizin- und Technikhistoriker eher häufiger biographisch arbeiten als ihre Kollegen in der Allgemeinhistorie, hat vielleicht auch damit zu tun, dass sich bei der Bearbeitung der Geschichte des eigenen Faches leichter eine gewisse Personalisierung des Zugangs einstellt als bei ferner liegenden Gegenständen. ${ }^{2}$ Dass weiterhin massenhaft anspruchslose Biographik produziert wird, liegt schließlich auch daran, dass Biographien sehr häufig außerhalb des Fachs geschrieben und gelesen werden. Professionelle Wissenschaftshistorie ist nur eine Variante unter zahlreichen Annäherungen an ihre historischen Gegenstände, und das wird kaum woanders so deutlich wie in der Biographik. Hier begegnen auch die Wissenschaftshistoriker und Wissenschaftshistorikerinnen ihrem weiteren Publikum, und die sich am methodischen Diskurs des Faches orientierenden Biographien konkurrieren somit mit all den anderen, die sonst noch geschrieben werden. ${ }^{3}$

Dennoch wird man der Diagnose Ludmilla Jordanovas aus dem Jahre 1993, „even for the really big names celebrated by whig history, few have been the recipients of sustained biographical treatment" (Jordanova 1993: 438), fünfzehn Jahre später nicht mehr zustimmen können. Stattdessen gibt es ein steigendes Interesse seitens professioneller Wissenschafts-, Medizinund Technikhistorikerinnen und -historiker zu konstatieren. Seit den 1990er Jahren ist eine Reihe von Biographien erschienen, die sich anspruchsvoller Methodik bedienen und in denen sich zeigt, dass die Biographik auf dem methodischen Niveau der übrigen Historiographie angekommen ist. ${ }^{4}$ Es war vor allem die seit einiger Zeit populäre mikrohistorische, epistemologisch interessierte Wissenschaftsgeschichte, die den biographisch Arbeitenden neue Perspektiven eröffnete. Zwar stellt sich mit dem Blick auf lokale Praktiken und ihre Akteure eine biographische Perspektive nicht von selbst ein, aber ein Text wie Frederick Holmes The Fine Structure of Scientific Creativity (Holmes 1981) eröffnete Perspektiven, die zunehmend genutzt wurden. Das zeigt sich zum Beispiel darin, dass die Zeitschrift ISIS sich des Themas 1996 in einem Schwerpunktheft annahm und dass eine aktuelle Einführung in die Medizingeschichte tatsächlich ein eigenes Kapitel zum Thema Biographie und Prosopographie enthält (Eckart/Jütte 2007: 219-229). Erwähnenswert sind auch Forschungen über scientific personae, also Persönlichkeitstypen, die zwischen individueller Lebensführung und gesellschaftlichen Institutionen vermitteln (Daston/Sibum 2003). Auch fehlt es nicht an anspruchsvollen Sammelwerken. Aus dem Arbeitsgebiet des Autors, der Medizingeschichte, seien hier für die internationale Forschung das Dictionary of Medical Biography und für die deutschsprachige Peter Voswinkels Weiterführung von Alfons Fischers Biographischem Lexikon der hervorragenden Ärzte der letzten fünfzig Jahre von 1932 genannt. $^{5}$ 
Insofern ist die Klage über das fehlende Ansehen der Biographik im Grunde das einzige, was an dem von Thomas Söderqvist herausgegebenen Sammelband The History and Poetics of Scientific Biography nicht überzeugt. ${ }^{6}$ Ansonsten aber ist es dem Herausgeber gelungen, die Autorinnen und Autoren, die allesamt über Erfahrungen als Biographen verfügen, zum Nachdenken über Form und Geschichte des Genres anzuregen. Es ist vor allem letzteres, was den Band besonders auszeichnet, und die verschiedenen Beiträge fügen sich zu einer kleinen Geschichte der Biographik in der Wissenschaftsgeschichte seit der Antike. Libia Taub etwa analysiert am Beispiel antiker Lebensbeschreibungen des Pythagoras, sogenannter Bioi, die scientific personae einer Epoche, in der die Eingebundenheit in Lehrer-Schüler-Verhältnisse die historische Persönlichkeit maßgeblich bestimmte. Gleich mehrere Autoren thematisieren den Aufstieg der Wissenschaftlerbiographie seit der Frühen Neuzeit. Descartes und Bacon entwarfen einen Philosophen-Typus, der sich wissenschaftlicher Fähigkeiten bediente, um eine reformierte Philosophie zu befördern. Im Zeitalter der Romantik wurden solche scientific personae dann zunehmend als geniale Einzelindividuen verstanden, was in der Folge das Verständnis wissenschaftlicher Arbeit wie auch das des Wissenschaftlers entscheidend prägte. Originell in der Wahl des Themas ist auch der Beitrag von Thomas Hankins, der das Aufkommen der Wissenschaftlerbiographie im 19. Jahrhundert mit der Entwicklung des Patentwesens in Beziehung setzt. In beiden Phänomenen zeige sich eine zunehmend individualisierte - materielle wie immaterielle - Gratifikation wissenschaftlicher Arbeit. Zu einer weit ausgreifenden und überdies sehr schwungvoll geschriebenen Verteidigung des Genres holt schließlich Jacalyn Duffin aus. Der Begriff der Biographie beschränke vielleicht den Gegenstand, gewiss aber nicht die Methode, erinnert sie ihre Leser: „We need to maximise our approaches, accommodate all genres - even if it means including the venerable and popular - if we really wish to understand our medical past." Gerade der Publikumsgängigkeit der Biographie wegen zählt sie hierzu ausdrücklich auch die Ästhetik der Form. Dass das beim Publikum so beliebte Genre von den Fachleuten lange stiefmütterlich behandelt wurde, verdanke sich nicht alleine skeptischen Verlegern, von denen Duffin manches zu erzählen weiß, sondern auch einer Distanz der Fachleute zur Biographie, in der sich mehr als nur ein Körnchen Snobismus in der Einstellung zum eigenen Publikum offenbare.

Verglichen mit Söderqvist verfolgt Peter Zigman mit seinem Band Die biographische Spur in der Kultur- und Wissenschaftsgeschichte bescheidenere Ziele. Ausgehend von der in der Einleitung konstatierten Renaissance des biographischen Zugangs in der Wissenschaftsgeschichte geht es ihm darum, die besondere Qualität dieser biographischen Spur aufzuzeigen. Sieht man einmal von dem etwas rätselhaften Zusatz „Kulturgeschichte“ ab, der im Band nicht eingelöst wird, so finden sich hier durchgehend interessante Arbeiten im weiteren Sinne wissenschaftshistorischen Zuschnitts. So etwa 
mehrere Beiträge, in welchen die Bearbeitungsgänge biographischer Sammelwerke und der darin aufgeführten Wissenschaftlerlemmata beschrieben werden. Reizvoll ist dies nicht zuletzt deswegen, weil der Herausgeber eine ganze Anzahl slowakischer und tschechischer Autorinnen und Autoren als Beitragende gewonnen hat und man so einiges über prosopographische und lexikalische Projekte in diesen Ländern erfährt, das einem sonst sicher entgangen wäre. Allerdings zeugen die Aufsätze dieses Bandes von einem unterschiedlich ausgeprägten Methodenbewusstsein, und mitunter vermisst man ein wenig die Spur der editorischen Arbeit in den Texten. Magdalena Hykšová beispielsweise beschränkt sich in ihren Methodological Notes on the Evaluation of Scientific Work de facto auf praktische Überlegungen zum Nutzen von Schautafeln in Wissenschaftlerbiographien und verzichtet im Übrigen auf jegliche methodische Reflexion. Andere Beiträge sind derart durch gewisse, rezenten Methodendiskursen geschuldete Dialekte geprägt, dass der mit feministischer Autobiographik nicht vertraute Rezensent doch ein wenig ins Schwimmen geriet. Als Zusammenfassung ihres Beitrages bietet Ertela Farkašovă etwa den folgenden, schwer verständlichen Satz an: „[...] the non identical/ and non-referential character of the auto-/biography doesn't imply the total absence to speak of the real self". Problem ist hier weniger das in einem Beitrag geforderte Methodenbewusstsein und auch nicht unbedingt der schlichte Zugang zum biographischen Material in anderen Aufsätzen, sondern dass kaum ein Zusammenhang zwischen den Beiträgen erkennbar ist. Sehr anregend ist dagegen der Aufsatz des Herausgebers, der sich anhand eines Tagebuches Gedanken über die Heuristik dieser in Biographien so beliebten Quellengattung macht. Von einem hermeneutischen Ansatz aus untersucht er anschaulich und geistreich den Übergang vom Selbst der autobiographischen Quelle zum Anderen des biographisch-deutenden Zugriffs darauf. Ebenfalls sehr lesenswert ist Carsten Reinhardts Text, der am Beispiel der von ihm und Tony Travis gemeinsam untersuchen Biographie des Chemikers Heinrich Caro zeigt, dass auch jenseits der Bourdieu'schen Kritik des Anscheins der Kohärenz in Biographien genügend Anlass besteht, personale Strukturen in historischen Prozessen zu untersuchen. Caros Ansichten etwa zur Eingebundenheit von Erfindern in gesellschaftliche und industrielle Strukturen sind einerseits Reflexe ihres Zeitalters, andererseits ermöglichen sie es zu verstehen, wie im 19. Jahrhundert industrielle Forschung in der chemischen Industrie nicht zuletzt von historischen Individuen mit ihren Vorstellungen und Werthaltungen gestaltet wurde.

Vor dem Hintergrund des aus den zwei Sammelbänden ersichtlichen Diskussionsstands lassen sich drei neuere Biographien lesen. Da ist zunächst Derek Lintons Emil von Behring. Infectious Disease, Immunology, Serum Therapy, ein Buch, das in eine veritable Lücke stößt. Eine nennenswerte englischsprachige Biographie Behrings existierte bislang nicht und selbst der des Deutschen mächtige Leser blieb auf der 1940 von dem Rassenhygieniker und 
Geomediziner Heinz Zeiss gemeinsam mit Richard Bieling veröffentlichen Biographie sitzen (Zeiss/Bieling, 1940). Bei allem Quellenreichtum war hier vor allem das Verhältnis zum Kollegen und Konkurrenten Paul Ehrlich in zeittypischer Weise und sehr unbefriedigend beleuchtet. Hinzu kam die erkennbare und problematische Tendenz der Autoren, Behring als zeitgemäße Führerpersönlichkeit zu begreifen.

Gemeinsam mit Sibasaburo Kitasato ist Behring in der Medizingeschichte vor allem als Erfinder des Diphtherieheilserums bekannt, das ab 1894 vermarktet wurde. Andere seiner Arbeiten wie die über das Tetanusserum sind weniger bekannt, erlangten aber im Ersten Weltkrieg einige Bedeutung. Nach einer spannungsgeladenen Zusammenarbeit mit der Firma Hoechst versuchte sich Behring auch selbst als Unternehmer und gründete die nach ihm benannte chemisch-phamazeutische Fabrik bei Marburg. Behrings Karriere ist zudem wie auch die seines Lehrers Robert Koch als Teil jenes ,Systems Althoff' gedeutet worden, in dem der allmächtige Ministerialdirektor im Preußischen Kultusministerium im fin de siecle gezielt Verbindungen zwischen chemisch-pharmazeutischer Industrie und universitärer Forschung förderte (Brocke 1991). Bekannt sind schließlich auch Klagen von Zeitgenossen über Behrings herrschsüchtiges Temperament, die ein reizvolles Problem für Biographen darstellen dürften.

Was fügt Linton diesem Bild hinzu? Leider nicht allzu viel. Die Biographie ist offenbar aus einem Projekt der Übersetzung wichtiger Texte Behrings entstanden (sie finden sich im Anhang). Das ist verdienstvoll, aber man gewinnt den Eindruck, dass der Autor sich nicht allzu weit von diesem Ansatz entfernt hat. In manchmal ermüdender Ausführlichkeit werden Behrings Arbeitsgebiete anhand seiner Publikationen referiert. Die Größe dieses Forschers, so die schlichte Grundthese Lintons, ist im Vergleich zu der Kochs oder Ehrlichs bislang nicht ausreichend gewürdigt worden. Gerade angesichts der zahllosen Kollegenkonflikte, in die Behring verwickelt war, reicht es aber nicht aus, wiederholt zu behaupten, dass er zumeist Recht hatte. Welche Einblicke man damit verschenkt sei an einem Beispiel verdeutlicht: Die Konflikte mit Paul Ehrlich entzündeten sich an der Frage des geistigen (und sonstigen) Eigentums am Diphtherieheilserum. Der Hintergrund war das von Ehrlich entwickelte Verfahren zur Wirksamkeitsmessung, die sogenannte Wertbestimmung. Behring bestritt weniger die Methode als solche als die damit verbundenen Geltungsansprüche. Für ihn war die Wertbestimmung ein Verfahren im Kontext der Herstellung des Diphtherieheilserums, für Ehrlich war sie nicht nur auf alle Heilseren, sondern im Grunde auf alle biologischen Heilmittel anwendbar. Eine solche Sicht der Dinge stärkte seine Position als Leiter der mit der entsprechenden Prüfung betrauten Reichsbehörde, des heutigen Paul-Ehrlich-Instituts, gegenüber dem auf seine Unabhängigkeit bedachten Behring. Dies ist der bei Linton fehlende Hintergrund der Behring'schen Kritik an Ehrlichs immunologischen Theorien (Hardy 2006). 
Dass Linton Behrings wissenschaftliche Arbeiten in verständlicher Sprache referiert, ist verdienstvoll, die Beschränkung auf diesen Ansatz macht die Biographie aber recht einseitig: So kommen Behrings Rolle als Unternehmer und die industrielle Seite der Serumtherapie insgesamt zu kurz. Das ist auch das Resultat der unbefriedigenden Quellengrundlage. Während Linton durch Nutzung des (in den USA lagernden) Nachlasses von Paul Ehrlich auf eine gute Quellengrundlage zum Verhältnis der beiden zugreifen kann, hat er auf den Besuch deutscher Archive verzichtet. ${ }^{7}$ Eine derartige Auslassung wird auch durch ihr Eingeständnis nicht besser. Der Autor hat sich damit um die Chance gebracht, über Behrings Verhältnis zu Staat, Industrie und Militär Neues zu Tage zu fördern. Außerdem bleibt er damit, abgesehen von seinen Recherchen in Ehrlichs Nachlass, auf dem Werk von Zeiss und Bieling (1940) als Quellengrundlage sitzen.

In der Summe verleitet das Bemühen, Behrings Ruf aufzupolieren den Autor zu enervierender Apologetik. Das erklärte Ziel, eine nach den methodischen Maßstäben unserer Zeit altmodische Biographie schreiben zu wollen (S. 15, Fn. 23), ist alleine noch keine Rechtfertigung eines solchen Unterfangens. Was soll man schließlich von einem Biographen halten, der in Abgrenzung zu Söderqvist erklärt, sein Buch sei keine Psychobiographie (S. 15) - nachdem er eine Seite zuvor festgestellt hat, „[t]oday, he [Behring; C.G.] would undoubtedly be diagnosed as suffering from manic depression (bipolar disorder)"?

Linton huldigt also der biographischen Illusion und er steht damit für eine mächtige Tradition heroisierender und leider zumeist anspruchsloser Biographik. Ihr vielleicht wichigstes Refugium sind, wie bereits erwähnt, historische Beiträge in naturwissenschaftlichen und medizinischen Fachzeitschriften. Hier werden die Pioniere jener Fächer noch so gefeiert, als habe die neuere Wissenschaftsgeschichte gar nicht stattgefunden, und es wimmelt in schönster Manier von Erfindern, Entdeckern und Helden (Schlich 1995). Die schiere Existenz einer Zeitschrift wie des Journal of Medical Biography verdankt sich, wie Jacalyn Duffin vermutet (2007: 189), möglicherweise dem Umstand, dass sich dort Texte abdrucken lassen, die andernorts kaum Chancen auf Veröffentlichung hätten.

Dass es auch anders geht, zeigen gleich zwei Biographien über den Psychoanalytiker und Zeitkritiker Alexander Mitscherlich, die 2007 in seinem 100. Geburtsjahr veröffentlicht wurden. Bekannt wurde Mitscherlich auf dreierlei Weise: als Berichterstatter der deutschen Ärzteschaft vom Nürnberger Ärzteprozess und Mitautor der daraus hervorgegangenen Dokumentation Medizin ohne Menschlichkeit; als Leiter des Frankfurter Sigmund Freud Institutes, von dem aus die westdeutsche Psychoanalyse nach 1945 wieder Anschluss an die internationale psychoanalytische Bewegung fand, und schließlich als sozialpsychologischer Zeitkritiker, dessen Schlagworte von der ,vaterlosen Gesellschaft', der ,Unfähigkeit zu Trauern’ oder der ,Un- 
wirtlichkeit unserer Städte' in den Wortschatz seiner Zeitgenossen eingingen. Es war vor allem letzteres, was Mitscherlichs Ruhm begründete, ein Ruhm, der zugleich eigentümlich begrenzt blieb. Die von Mitscherlich als Synthese aus Soziologie und Psychologie gedachten Analysen erschienen Mitte der 1960er Jahre weithin plausibel, aber zehn Jahre später löste sich das mimetische Verhältnis zur Öffentlichkeit bereits wieder auf. Im Unterschied zum Ansehen etwa Jürgen Habermas' und anderer intellektueller Ikonen des gesellschaftlichen Aufbruchs der 1968er blieb Mitscherlichs Popularität eine Mode ihrer Zeit.

Beide Bücher gehen auf recht unterschiedliche Weise mit dieser Ausgangslage um. Martin Dehli bricht schlicht vor dem Ruhm ab und erzählt die Geschichte einer Selbsterfindung seit den späten 1920er Jahren. Sein Mitscherlich ist ein Suchender, der aus einem konfliktgeladenen Vater-SohnVerhältnis kommend sich an immer neuen Vaterfiguren abarbeitet und stets mit diesen brechend zu der intellektuellen Prägnanz findet, die ihn schließlich in den 1960er Jahren auszeichnete. Dass die erste dieser Vaterfiguren Ernst Jünger hieß und Mitscherlich sich bis weit in die NS-Zeit hinein im intellektuellen Milieu der konservativen Revolution bewegte, dies präzise herausgearbeitet zu haben, gehört zu den Verdiensten von Dehlis Biographie. Von der Distanz zum Faschismus bis zur Bejahung der Demokratie war es ein weiter Weg. Mitscherlich unterschlug dies in seiner Autobiographie nicht, beschönigte es aber doch in mancher Hinsicht (Mitscherlich 1980). Erst zum Kriegsende hin kam der Prozess des politischen Umdenkens zum Abschluss, und es ist in diesem Sinne nicht überraschend, dass Mitscherlich einen Demokraten wie den ebenfalls in Heidelberg lebenden Nationalökonomen Alfred Weber erst nach Kriegsende kennenlernte. Die intellektuelle und institutionelle Heimat jener Jahre - und die nächste Vaterfigur - lieferte ihm die sogenannte biographische Medizin Victor von Weizsäckers. Was die Psychologie betraf, so hatte Mitscherlich zwar schon in den späten 1930er Jahren Kontakte zu Psychoanalytikern in der Schweiz geknüpft, gab aber erst nach Kriegsende die biographische Medizin Weizsäckers zugunsten der Psychoanalyse Freuds auf. Man nimmt Mitscherlich nichts, wenn man mit Dehli feststellt, dass er die Neigung hatte, Lernprozesse wie zum Beispiel seinen Antifaschismus oder seine Hinwendung zur Psychoanalyse retrospektiv zu projizieren. Es ist vielmehr für den Leser sehr eindrucksvoll, diesen Lernprozess mitzuverfolgen, der schließlich unter anderem in Mitscherlichs Bereitschaft kulminierte, vom Nürnberger Ärzteprozess zu berichten - wohl wissend, dass er sich damit die erbitterte Gegnerschaft eines großen Teils der deutschen Ärzteschaft einhandeln würde. ${ }^{8}$

Deutlich wird auch, mit welchem Geschick Mitscherlich in den ersten Jahrzehnten der Bundesrepublik die Kombination von fachlichem und politischem Außenseitertum zu nutzen wusste, um sich - auch gegenüber dem Ausland - als zeitgemäßer, weil unbelasteter Sachwalter eines politisch bela- 
steten Faches zu positionieren. Gerade was die Psychoanalyse betraf, dominierte aber auf die Dauer der Politiker den Arzt: Seine klinischen Arbeiten in Heidelberg blieben ohne größere Resonanz, als Direktor des Frankfurter Sigmund-Freud-Institutes aber wurde Mitscherlich zum Sprachrohr und Repräsentanten der westdeutschen Psychoanalyse. Dieses und mehr erfährt man in Dehlis gut recherchiertem und geschriebenem Buch, dem man die Neugier auf den Untersuchungsgegenstand deutlich anmerkt. Auch methodisch ist das Buch auf der Höhe der Zeit. Es zeichnet das Bild Mitscherlichs als einer ausgesprochen vielschichtigen und getriebenen Persönlichkeit.

Tobias Freimüllers im gleichen Verlag erschienene Biographie zeigt allerdings, dass bei Mitscherlich noch mehr zu holen ist. Hier wird die intellektuelle Biographie Mitscherlichs in ihrer Gesamtheit in den Blick genommen. Freimüller bettet ihn deutlich mehr in die Kulturgeschichte des Zeitalters ein als Dehli und geht von Mitscherlichs faszinierender Popularität in den 1960er und 1970er Jahren aus. Die Betrachtung dieses Phänomens trägt jenseits der Person des Psychoanalytikers auch viel zum Verständnis der Aufbruchsstimmung jener Jahre bei. Das schließt nicht aus, dass Freimüller an Dehli anknüpft, was er im Gegenteil ausdrücklich tut. Vielmehr ist die veränderte Perspektive eine Folge der Ausweitung des Untersuchungszeitraumes über die frühen 1960er Jahre hinaus. Dehlis Mitscherlich ist im Wesentlichen ein politisch denkender Arzt, bei Freimüller tritt der charismatische Feuilletonist in den Vordergrund, der der Gesellschaft im Aufbruch die Stichworte lieferte. Seine Kritiken eröffneten Wege, der deutschen Geschichte ins Auge zu blicken und sich gleichzeitig von ihr zu distanzieren. Wie Freimüller sehr überzeugend herausarbeitet, lieferte Mitscherlich mit seinen Analysen wichtige Beiträge zur Selbstbegründung der bundesdeutschen Nachkriegsgesellschaft als einer demokratischen. Die gelegentlichen Untiefen seiner Analysen standen ihrer Popularität nicht im Wege, vielmehr war es gerade das milde Licht psychologischer Sublimierung, das ihre Wirkung begründete und bemerkenswerte Auslassungen ermöglichte. So etwa die, dass in der Analyse der vaterlosen Gesellschaft es stets um den Übervater Adolf Hitler geht, der Verlust einer ganzen Generation von Vätern im Krieg hingegen mit keinem Wort erwähnt wird.

„[... in dieser Doppeldeutigkeit und Unschärfe lag die Attraktivität der Diagnose begründet. Sie thematisierte einen historischen Tatbestand [der NS-Vergangenheit; CG], dem auch 1967 noch hohe Brisanz innewohnte, und ermöglichte es dennoch, die Frage nach historischer Schuld und Verantwortung zu umgehen. In der Kollektivpsychologie verschwand die historische Realität, kaum dass sie in zahlreichen skandalösen personellen Kontinuitäten und Skandalen die westdeutsche Öffentlichkeit erschüttert hatte." (S. 433)

Von dieser eigentümlichen Beschaffenheit aus erklärt Freimüller die sonderbare Folgenlosigkeit der Mitscherlich'schen Analysen. Jenseits ihres nicht selten pompösen Begriffsapparates waren sie oft oberflächlich. Die angestrebte Synthese von Soziologie und Psychologie blieb eine Art Papiertiger 
und erwies sich bereits im eigenen Sigmund-Freud-Institut als undurchführbar. Auch die anfängliche Begeisterung anderer Intellektueller wie etwa Max Horkheimer für Mitscherlich wich bald der Erkenntnis, dass es seinem Denken an Tiefe und theoretischer Stringenz durchaus mangelte.

Dass der 1908 geborene Mitscherlich selbst ein Exponent jener vaterlosen Generation war, belegt einen eindrucksvollen persönlichen Lernprozess. Dieser führte seinen Protagonisten von der konservativen Revolution in die 1968er Bewegung und vom raunenden Existenzialismus Weizsäckers zu Freuds Psychoanalyse. Freimüller formuliert auf dieser Basis eine These, die einen solchen, von der Neigung zu radikalen Brüchen und zur weltanschaulichen Aufladung gekennzeichneten Lebensweg überzeugend in den Rahmen der deutschen Geschichte einbindet. In Habitus und Lebensalter hatte Mitscherlich vieles mit jener "Generation des Unbedingten" (Wildt 2002) gemeinsam, der zum Beispiel das Führungspersonal des Reichssicherheitshauptamtes entstammte. Mit deren Protagonisten teile er, so Freimüller, die Erfahrung extremer Instabilität, das Misstrauen in die Institutionen bürgerlicher Demokratie und die Neigung zu einer Form vehementer Kritik, bei der die Radikalität der Durchführung oft als Rechfertigung des Unterfangens selbst erschien. Es waren die radikalen Brüche, die Mitscherlichs Leben kennzeichneten: mit dem Vater, mit Ernst Jünger, mit der biographischen Medizin Weizsäckers, mit dem Analytiker Felix Schottländer in den 1950er Jahren. Dass er sich bei all dem als lernfähig erwies, war ein Glück für ihn und für die Gesellschaft, in der er lebte, und lässt ihn gewissermaßen als positiven Gegenentwurf zur jüngeren Führungsgeneration des NS-Staates erscheinen. Freimüller ist in diesem Sinne auch methodisch etwas sehr Eindruckvolles gelungen: unter ausdrücklichem Bezug auf Bourdieus Kritik der Kohärenzillusion der Biographik die Geschichte einer sich stets neu erfindenden historischen Persönlichkeit zu schreiben.

Nimmt man die fünf besprochenen Titel als Indiz für das, was Biographik in der Wissenschaftsgeschichte zu leisten vermag, so ist die Lage insgesamt erfreulich. Das Genre hat als Sündenbock anspruchsloser Geschichtsschreibung ausgedient. Dafür gibt es schlicht und einfach zu viele überzeugende Biographien, die methodisch auf dem Niveau ihrer Zeit sind. Auch hat sich, wie eingangs erwähnt, mittlerweile ein historiographischer Diskurs entwickelt, der denjenigen, die sich an ein solches Unternehmen machen, als Orientierung dienen kann. Dass ein Leben allein noch keine Biographie ist, sondern sich diese in der historischen Rekonstruktion erst herstellt, hat sich mittlerweile herumgesprochen. Dass gleichzeitig weiterhin auch mäßig recherchierte, methodisch unbefriedigende Biographien veröffentlicht werden, widerspricht diesem Befund nicht unbedingt. Vielmehr illustriert dieser Umstand ein Grundproblem der Geschichte der Medizin, Naturwissenschaft und Technik: Das öffentliche Interesse an ihren Themen ist groß, aber die Bereitschaft, in ihre Erforschung zu investieren, bleibt alles in allem 
gering. Und so gibt es in Deutschlands Gewerbegebieten zahllose Straßen, die nach Männern und Frauen aus der Wissenschaft, Technik und Medizin benannt sind. Gleichzeitig hält sich Zahl der brauchbaren Biographien der solchermaßen auf Blech gebannten historischen Persönlichkeiten in überschaubaren Grenzen.

\section{Anmerkungen}

1 Stern formulierte in seiner Einleitung eine deutliche Kritik einer das historische Individuum vernachlässigenden Strukturgeschichte.

2 Lutz Raphael hat diese Beobachtung für die Historiographiegeschichte formuliert (Raphael 1996).

3 Erkennbar ist auch eine gewisse Metaphorisierung des Begriffes zu beobachten, wenn etwa Biographien von Städten, Krankheiten oder ganzer Kontinente veröffentlicht werden.

4 Man denke an Autoren wie Adrian Desmond und James More (Darwin), Theodore Porter (Pearson), Gerald Geison (Pasteur), Frederic Holmes (Krebs), Constantin Goschler (Virchow) oder Thomas Söderqvist (Jerne). Als Einführung eignen sich Focus. Biography in the History of Science, in dem die Zeitschrift Isis 1996 mehrere Beiträge zusammenfasste, sowie die Sammelbände Füßl/Ittner 1997 und Shortland/Yeo 1996.

5 Von Voswinckel 2002 ist leider bislang nur der erste Band erschienen; am Dictionary of Medical Biography war der Verfasser dieses Beitrags als Autor und Teilherausgeber beteiligt.

6 Söderqvist ist der Verfasser einer Biographie des Dänischen Immunologen Niels Jerne, die mittlerweile in englischer Übersetzung vorliegt (Söderqvist 2003 [1998]).

7 Die im Anhang zitierten „Dokumente aus Hoechster Archiven“ finden sich bekanntlich auch in Bibliotheken. Das im Verzeichnis genannte Archiv „Hoechst“ heißt nach der Auflösung der Hoechst AG bereits seit 1999 Histocom.

8 Seine Familie hat das offenbar anders gesehen. Sie hintertrieb erfolgreich einen bereits mit einem Frankfurter Verlag geschlossenen Vertrag für Dehlis Manuskript. Dies ist die Erklärung dafür, warum der Wallstein Verlag gleich zwei Biographien Mitscherlichs im selben Jahr veröffentlichen konnte (Brumlik 2008).

\section{Literatur}

Boedeker, Hans Erich, Hg., 2003. Biographie schreiben. Göttingen: Wallstein.

Bourdieu, Pierre, 1990 [1986]. Die biographische Illusion. BIOS. Zeitschrift für Biographieforschung und Oral History, 75-81 [La illusion biographique, Actes de la recherche en sciences sociales, 62/63, 69-72].

Brocke, Bernhard vom, Hg., 1991. Wissenschaftsgeschichte und Wissenschaftspolitik im Industriezeitalter. Das „System Althoff" in historischer Perspektive. Hildesheim: Lax.

Brumlik, Micha, 2008. Der Große Alexander. Die Zeit, 18. September 2008.

Daston, Lorraine/Sibum, H. Otto, Hg., 2003. Scientific Personae and Their Histories. Science in Context, 16, 1/2.

Duffin, Jacalyn, 2007. ,La Mauvaise Herbe؛. Unwanted Biographies Both Great and Small. In Thomas Söderqvist, Hg., The History and Poetics of Scientific Biography. Aldershot: Ashgate, 185-197.

Eckart, Wolfgang U./Jütte, Robert, 2007. Medizingeschichte. Eine Einführung. Köln/Weimar/ Wien: Böhlau.

Füßl, Wilhelm/Ittner, Stefan, 1997. Biographien und Technikgeschichte. Leverkusen: Leske + Budrich. 
Hankins, Thomas, 1979. In Defence of Biography. The Use of Biography in the History of Science. History of Science, 17, 1-16.

Hardy, Anne I., 2006. Paul Ehrlich und die Serumproduzenten. Medizinhistorisches Journal, 41, 51-84.

Holmes, Frederic L., 1981. The Fine Structure of Scientific Creativity. History of Science, 19, 60-70.

Jordanova, Ludmilla, 1993. Has the Social History of Medicine Come of Age? The Historical Journal, 36, 437-449.

Klein, Christian, Hg., 2002. Grundlagen der Biographik. Theorie und Praxis des biographischen Schreibens. Stuttgart: Metzler.

Merton, Robert K., 1985. Prioritätsstreitigkeiten in der Wissenschaft. In: Ders., Entwicklung und Wandel von Forschungsinteressen. Frankfurt: Suhrkamp, 258-300.

Mitscherlich, Alexander, 1980. Ein Leben für die Psychoanalyse. Anmerkungen zu meiner Zeit. Frankfurt: Suhrkamp.

Müller, Burkhard, 2008. Gut gefälscht ist halb gewonnen. Süddeutsche Zeitung, 17. März 2008.

Oelkers, Jürgen, 1974. Biographik. Überlegungen zu einer unschuldigen Gattung. Neue Politische Literatur, 19, 296-309.

Raphael, Lutz, 1996. Diskurse, Lebenswelten und Felder. Implizite Vorannahmen über das soziale Handeln von Kulturproduzenten im 19. und 20. Jahrhundert. In: Wolfgang Hartwig und Hans-Ulrich Wehler, Hg., Kulturgeschichte heute. Geschichte und Gesellschaft, 16 (Sonderheft), 165-181.

Schlich, Thomas, 1995. How Gods and Saints Became Transplant Surgeons. The Scientific Article as a Model for the Writing of History. History of Science, 33, 31-331.

Shortland, Michael/Yeo, Richard, Hg., 1996. Telling Lives in Science. Essays on Scientific Biography. Cambridge: Cambridge University Press.

Sombart, Nikolaus, 1978. Der letzte Kaiser war so, wie die Deutschen waren. Wilhelm II. Vergangenheitsbewältigung und Wiedergutmachung. Frankfurter Allgemeine Zeitung, 27. Januar 1978 .

Söderqvist, Thomas, 2003 [1998]. Science as Autobiography. The Troubled Life of Niels Jerne. New Haven: Yale University Press.

Stern, Fritz, 1988 [1977]. Gold und Eisen. Bismarck und sein Bankier Bleichröder. Reinbek: Rowohlt.

Voswinckel, Peter, 2002. Biographisches Lexikon der hervorragenden Ärzte der letzten fünfzig Jahre von Isidor Fischer, Berlin und Wien 1932-1933, Nachträge und Ergänzungen. Hildesheim: Olms.

Wildt, Michael, 2002. Generation des Unbedingten. Das Führungskorps des Reichssicherheitshauptamtes. Hamburg: Hamburger Edition.

Zeiss, Heinz/Bieling, Richard, 1940. Behring. Gestalt und Werk. Berlin: Bruno Schultz Verlag.

\section{Christoph Gradmann}

Institute of General Practice and Community Medicine

Section for Medical Anthropology and Medical History

University of Oslo

P.O.Box 1130 Blindern

$\mathrm{N}-0318$ Oslo

E-Mail: Christoph.Gradmann@medisin.uio.no 